\title{
2D Tomographic Imaging of the Edge Turbulence in RFX-Mod
}

\author{
Gianluigi SERIANNI, Roberto CAVAZZANA, Matteo AGOSTINI ${ }^{1)}$ and Paolo SCARIN \\ Consorzio RFX, Associazione EURATOM-ENEA sulla fusione, C.so Stati Uniti 4 35127, Padova, Italy \\ 1) Dipartimento di Fisica, Università di Padova, Padova, Italy
}

(Received 9 December 2006 / Accepted 17 June 2007)

\begin{abstract}
In the reversed Field Pinch Experiment RFX-mod a Gas Puffing Imaging Diagnostic (GPID) is used to investigate the turbulence of the edge plasma. The system consists of a gas puffing nozzle and 32 optical channels to measure the HeI $(668 \mathrm{~nm})$ line emission. The lines of sight are arranged into three fans intersecting each other in an area normal to the main magnetic field. The diagnostic system provides an analogue bandwidth of $2 \mathrm{MHz}$ and all channels are simultaneously sampled at $10 \mathrm{MSamples} / \mathrm{s}$ for the whole discharge duration $(350 \mathrm{~ms})$. Different inversion techniques have been applied to the data in order to obtain a 2D tomographic reconstruction of the light emission pattern from the line integrals. Comparison shows that the most suitable method is based on 2D spatial Fourier expansion, applying the Singular Value Decomposition technique with regularisation. The high sampling time allows to obtain a 2D image every $100 \mathrm{~ns}$. It is found that emission structures ("blobs") emerge from the background turbulence; a characterisation is given by computing the energy of the Fourier modes.
\end{abstract}

(C) 2007 The Japan Society of Plasma Science and Nuclear Fusion Research

Keywords: edge turbulence, electrostatic structure, gas puff imaging, tomographic reconstruction, reversed field pinch

DOI: $10.1585 /$ prr.2.S1119

\section{Introduction}

In nuclear fusion devices confinement performances are strongly affected by the physics of plasma transport in the edge region [1]. Huge fluctuations are commonly found in the signals measured in the edge plasmas. Such events are usually interpreted as the signature of coherent structures moving within the plasma [2]. These are commonly recognised to be responsible for the radial transport of particles [3].

In order to visualise the coherent structures and to investigate their dynamics, a Gas Puff Imaging (GPI) system has been developed for the RFX-mod experiment [4]. The diagnostic collects the light emitted by the interaction of the plasma with a neutral gas puffed locally. Similar systems are used in tokamaks where the low curvature of magnetic field lines allows to use fast cameras [5]. Conversely, due to the unfavourable position of access ports, in the Reversed Field Pinch (RFP) experiment RFX-mod a tomographic-like arrangement of lines of sight has been adopted on a plane perpendicular to the main magnetic field.

The present paper is dedicated to the techniques adopted to analyse the experimental data given by the GPI system. In par. 2 a description of the diagnostic is given; in par. 3 the methods are introduced to invert the lineintegrated signals and obtain the local emissivity; finally in par. 4 the methods are tested on synthetic and experimental signals.

\section{Description of the Diagnostic}

The GPI consists of a gas-puffing nozzle, through which gas is puffed into the plasma edge, and an optical system. The equipment is thoroughly described in Ref. $[6,7]$; only a brief summary will be given here.

The nozzle is fed by a gas reservoir through a fast piezoelectric valve; the puffed gas is helium, whereas the main gas is hydrogen [7]. The optical system is made of 32 lines of sight (LoS), distributed into three fans (see Fig. 1). The $16 \mathrm{LoS}$ of fan no. 1 enter the plasma almost radially; two other fans (eight LoS each) after reflections

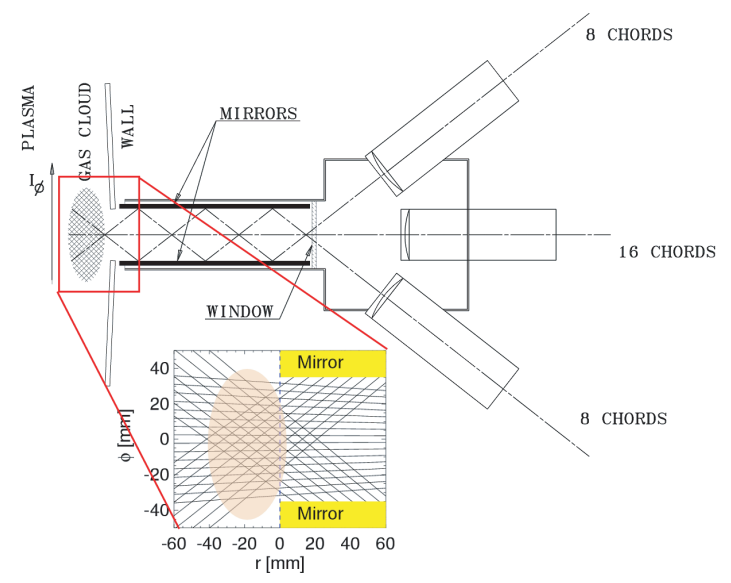

Fig. 1 Schematic drawing of the GPI system in the radialtoroidal plane, sketching the three fans of optical chords focused onto a puffed cloud; the inset shows a zoom of the region of the puffed gas cloud. 
on a pair of parallel mirrors. The three fans intersect in the puff region. The viewed plane is perpendicular to the local main magnetic field, which is poloidal in the plasma edge of RFPs. By means of optical fibres, the light reaches two 16-channel photomultiplier tubes (PMTs). The HeI line at $667.8 \mathrm{~nm}$ is selected by means of interference filters. The optical system is focused onto the viewing area of 70 (toroidal) $\times 4$ (poloidal) $\mathrm{mm}^{2}$ with a spatial resolution of $4.5 \mathrm{~mm}$ and a focal depth of $50 \mathrm{~mm}$ along the radial direction. The GPI is mounted at an external equatorial port of RFX-mod.

After trans-impedance amplifiers with $2 \mathrm{MHz}$ bandwidth, all 32 signals are recorded using a standalone data acquisition system having DC-8 MHz bandwidth with sampling interval of $100 \mathrm{~ns}$ and 4 Mwords of 12 bit resolution per channel.

\section{Reconstruction Algorithms}

The main task of the reconstruction technique is to recover the emissivity pattern in the toroidal-radial plane $e(r, z)$ from the measured line-integrated signals $l_{i}$.

The simplest method developed to obtain a rough image from the $32 \mathrm{LoS}$ measurements is an application of the back-projection algorithm [8].

From the inset of Fig. 1 it is clear that lines belonging to each of the three fans intersect in some points in the centre of the viewed plasma region. If $\left(r^{*}, z^{*}\right)$ are the coordinates of one of these points, the plasma emission can be written as $e\left(r^{*}, z^{*}\right) \propto l_{1} l_{2} l_{3}$, meaning that the signal at the intersection point is assumed to be proportional to the product of the three line integrals intersecting in $\left(r^{*}\right.$, $\left.z^{*}\right)$, because only the light emitted in the vicinity of the intersection point will give a contribution to all three LoS, yielding a triple product significantly different from zero.

Another way to solve the reconstruction problem consists in expanding the emissivity in a spatial Fourier series for each sampling time, according to the formula:

$$
\begin{aligned}
e(r, z)= & \sum_{k q}\left[C_{k q} \cos \left(\frac{k \pi z}{\Delta z}\right) \cos \left(\frac{q \pi r}{\Delta r}\right)+\right. \\
& +D_{k q} \sin \left(\frac{k \pi z}{\Delta z}\right) \cos \left(\frac{q \pi r}{\Delta r}\right)+ \\
& +F_{k q} \cos \left(\frac{k \pi z}{\Delta z}\right) \sin \left(\frac{q \pi r}{\Delta r}\right)+ \\
& \left.+G_{k q} \sin \left(\frac{k \pi z}{\Delta z}\right) \sin \left(\frac{q \pi r}{\Delta r}\right)\right]
\end{aligned}
$$

where $\Delta z$ and $\Delta r$ are respectively the toroidal and the radial dimensions of the edge region to which the inversion is applied, $k$ and $q$ are the toroidal and radial mode numbers, and the coefficients $C_{k q}, D_{k q}, F_{k q}$ and $G_{k q}$ are the unknowns to be determined. The unknowns $C_{k q}, D_{k q}$, $F_{k q}$ and $G_{k q}$ can be re-written as the components (in that order) of a vector $A_{j}$. Integrating Eq. 1 along the $32 \mathrm{LoS}$ produces the matrix equation $l_{i}=\sum_{j} W_{i j} A_{j}$, where the coefficients $W_{i j}$ are the result of the integration of the sine and cosine functions along the LoS. Previous experimental results concerning electrostatic structures [9] show that $k=0-5$ and $q=0-3$ should be sufficient to describe the structures in RFX-mod edge. Using this choice of toroidal and radial wave-numbers, with only 32 experimental LoS integrals, the resulting system of equations is underdetermined and it is not possible to invert the matrix $W$ exactly.

To estimate the unknown coefficients the Singular Value Decomposition (SVD) technique is applied [10,11]. The SVD allows solving the unconstrained linear least squares problem: $\min (J(\boldsymbol{A}))=\min \|\boldsymbol{I}-W \boldsymbol{A}\|_{2}$. Tikhonov regularisation has been adopted [12], with a penalty function depending on a parameter $\lambda$, aimed at reducing smallscale fluctuations, because, in this ill-posed problem, a small perturbation of $\boldsymbol{A}$ may lead to a large perturbation of the solution. The parameter $\lambda$ is chosen with the Lcurve technique [13-15], which is commonly used for the tomographic inversion problems in fusion plasma experiments [16].

\section{Application of the Methods}

To test both the back-projection and the tomographic inversion algorithm, an emission pattern has been simulated, the LoS integrals have been numerically evaluated, and the inversion algorithms have been applied.

Fig. 2 shows the simulation and the reconstruction results. A Gaussian emitting pattern has been located in the zone where the LoS intersect each other (Fig. 2 top left); at the bottom left the reconstruction using the Fourier series is shown, and on the right the back-projection result. Both inversion techniques place the centre of the Gaussian correctly in the toroidal-radial plane. Also the toroidal exten-

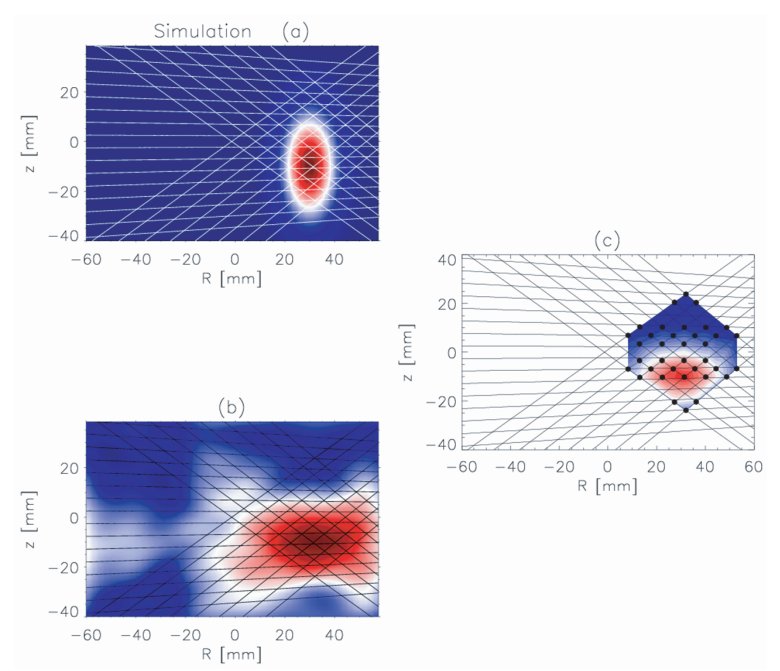

Fig. 2 Simulation of a Gaussian emitting pattern and results of reconstruction through back-projection (right) and Fourier development (bottom). The traces of the LoS are shown. In the right figure the black points represent the position where three lines of sight intersect each others. 


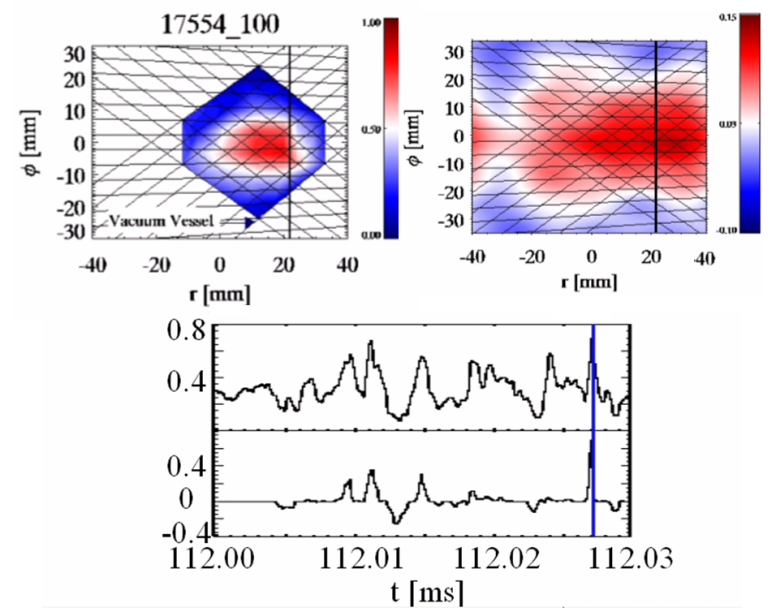

Fig. 3 2D inverted pattern of a coherent structure of the edge plasma. Top: reconstruction with back-projection (left) and with Fourier method (right). Bottom: time behaviour of one GPI signal and one of the triple products. The inversion is made at $t=101.0271 \mathrm{~ms}$.

sion of the reconstructed pattern is similar to the original one, indicating that the optical system used and the numerical algorithm allow a good toroidal resolution. The radial resolution is a more critical problem: the inverted pattern using the Fourier series shows a wider radial extension than the original one. This is probably due to the little inclination of the two lateral fans of LoS: the result is a broad structure elongated along the direction of these fans. An emitting region is also reconstructed around $r=-60 \mathrm{~mm}$ : this is an aliasing phenomenon since in that region there are no LoS intersections.

The reconstructed pattern with the back-projection technique has the opposite limitation, as the three-point intersections are limited to a small radial region.

In the following the application of the two inversion algorithms to the experimental data is described. The main aim is to detect the coherent structures that characterise the plasma edge and to study their motion and evolution.

For the 32 measured time series of integrated signals a time slice is selected to which the inversion is applied; the time average of each signal is computed and subtracted from the signal; the signals are subsequently divided by their average. These new time series are low-pass filtered at $1 \mathrm{MHz}$ to avoid noise problems that could make difficult the tomographic inversion. Then the two inversion algorithms are applied to the data to obtain a 2D imaging every sampling time, namely every $100 \mathrm{~ns}$.

In Fig. 3 an example of inversion is shown at time instant $t=101.0271 \mathrm{~ms}$. On top of the figure a comparison is provided between the two methods used: on the left the inversion using the back-projection method, and on the right using the Fourier expansion. In both graphs the black vertical line indicates the vacuum vessel position. In the lower

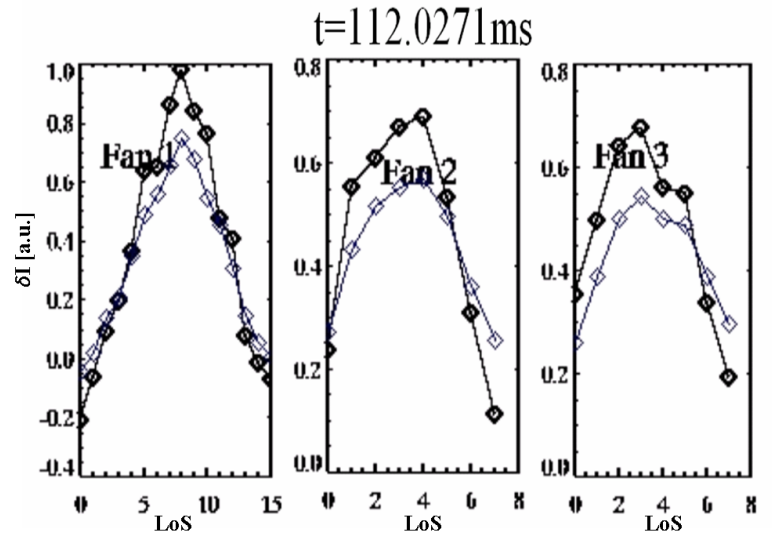

Fig. 4 Experimental (dark diamonds) and Fourier-series reconstructed (light diamonds) line integrals of the GPI for the three fans; same discharge and time as in Fig. 3.

graph, the top panel reports the time behaviour of one LoS signal; the bottom panel one of the triple products; the vertical line denotes the time used for the inversion displayed.

It is clear that the integrated emission possesses a peak; both methods can reconstruct a structure passing in front of the diagnostic field of view. For the time instant of Fig. 3, the inverted emissivity structure is centred at about $0 \mathrm{~mm}$ in the toroidal direction. The two reconstructed emissivity patterns differ because the tomographic reconstruction is stretched along the direction of the lateral fans, giving a poor radial resolution (Fig. 2). As anticipated, such observation is related to insufficient LoS intersections and to a little inclination of the lateral fans. On the other hand the toroidal resolution of both inversion methods is good.

The main difference between the two algorithms seems to be the dimension (especially radial) of the emissivity pattern: this is an intrinsic characteristic of the backprojection method. As it can be seen at the bottom of Fig. 3, the triple product signal in correspondence of a GPI peak is narrower than one of the original signals. The triple product is different from zero only when all three signals are simultaneously different from zero; consequently the structure reconstructed by back-projection results smaller than what is deduced from the time signals.

To verify the goodness of the reconstruction with the Fourier method, in Fig. 4 the line-integral signals measured by the GPI (dark diamonds) and those reconstructed by the Fourier algorithm (light diamonds) are compared; the reconstructed line integrals are computed by integrating the inverted emissivity along the $32 \mathrm{LoS}$. The reconstructed signals are not exactly superimposed to the experimental ones: using the L-curve regularisation method, the SVD inversion searches for a solution that minimises both the $\chi^{2}$ and the penalty function, which aims at a smooth $2 \mathrm{D}$ solution.

By applying the tomographic reconstruction to each 


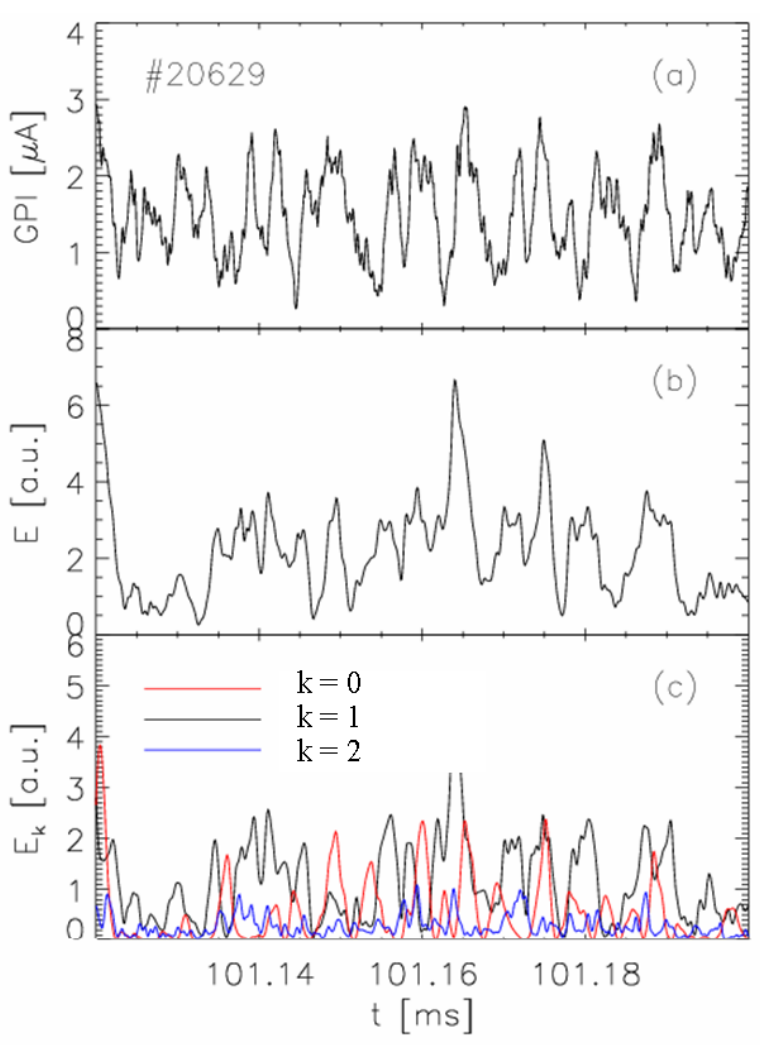

Fig. 5 Signal of one GPI LoS (a), total mode energy of the tomographic inversion (b), and energy of toroidal modes (c).

sampling time, information is gained concerning the analysis of the motion and the evolution of edge structures.

The inversion algorithm gives the values of the coefficients $C_{k q}, D_{k q}, F_{k q}$, and $G_{k q}$ as a function of time; they contain all the physical information of the image. Such coefficients can be interpreted as the amplitude of the modes with mode number $k$ and $q$ : so the time evolution of the total energy can be evaluated

$$
E(t)=\sum_{k q} C_{k q}^{2}(t)+D_{k q}^{2}(t)+F_{k q}^{2}(t)+G_{k q}^{2}(t)
$$

as well as the energy of a particular toroidal mode.

In Fig. 5 the time behaviour of the mode energies $(b, c)$ is compared with the GPI signal (a). The total mode energy $E(t)$ peaks in correspondence to peaks in the GPI signal, which in turn correspond to structures in the reconstructed images. In Fig. 5 (c) the time behaviour of different toroidal $k$ modes is shown: the two dominant toroidal modes are the $k=0$ and $k=1$.

\section{Conclusions}

In the present paper the techniques adopted to analyse the experimental data given by the GPI system in the Reversed Field Pinch experiment RFX are presented, to study the coherent structures in the plasma edge.

The reconstruction algorithms using back-projection and Fourier-series are compared, showing that the geometry of the lines of sight gives just some indications about the radial dimension of structures, whereas the toroidal patterns can be well diagnosed.

The Fourier-series expansion of the signals provides several tools to investigate the structures, such as the global mode energy and the energy of a single mode, which identify the presence of structures in the emission.

With the algorithms described herein, it can be stated that our realisation of the GPI diagnostic is a valid substitute of fast cameras, which could not be used where the curvature of magnetic field lines is unfavourable, such as in the RFP. For the sake of comparison, it should be added that the GPI requires only one access port and provides a very high time and space resolution as well as signal-tonoise ratio.

\section{Acknowledgements}

This work was supported by the European Communities under the contract of the Association between EURATOM and ENEA. The views and opinions expressed herein do not necessarily reflect those of European Commission.

[1] B.A. Carreras, IEEE Trans. Plasma Sci. 25, 1281 (1997).

[2] B.K. Jha et al., Phys. Rev. Lett. 69, 1375 (1992).

[3] V. Antoni et al., Phys. Rev. Lett. 87, 045001 (2001); M. Spolaore et al., Phys. Rev. Lett. 93, 215003 (2004).

[4] G. Rostagni, Fusion Eng. Des. 25, 301 (1995); P. Sonato et al., Fusion Eng. Des. 66-68, 161 (2003).

[5] R.J. Maqueda et al., Rev. Sci. Instrum. 72, 931 (2001); S.J. Zweben et al., Phys. Plasmas 9, 1981 (2002).

[6] R. Cavazzana et al., Rev. Sci. Instrum. 75, 4152 (2004).

[7] M. Agostini, R. Cavazzana, P. Scarin, G. Serianni, Rev. Sci. Instrum. 77, 10E513 (2006).

[8] F. Natterer, The mathematics of computerized tomography (Stuttgart, Teubner, 1986).

[9] V. Antoni et al., Phys. Rev. Lett. 79, 4814 (1997).

[10] P.C. Hansen, SIAM J. Sci. Statist. Comput. 11, 503 (1990).

[11] P.C. Hansen, SIAM J. Sci. Statist. Comput. 13, 1142 (1992).

[12] A.N. Tikhonov, Soviet. Math. Dokl. 4, 1035 (1963).

[13] P.C. Hansen, SIAM Review 34, 561 (1992).

[14] P.C. Hansen and D.P.O'Leary, SIAM J. Sci. Statist. Comput 14, 1487 (1993).

[15] F. Benyah and L.S. Jennings, J. Austral. Math. Soc. B 40(E), E138 (1998).

[16] P. Franz et al., Nucl. Fusion 41, 695 (2001). 